Document downloaded from:

http://hdl.handle.net/10251/80757

This paper must be cited as:

Broatch Jacobi, JA.; Guardiola, C.; Bares-Moreno, P.; Denia Guzmán, FD. (2016). Determination of the resonance response in an engine cylinder with a bowl-in-piston geometry by the finite element method for inferring the trapped mass. International Journal of Engine Research. 17(5):534-542. doi:10.1177/1468087415589701.

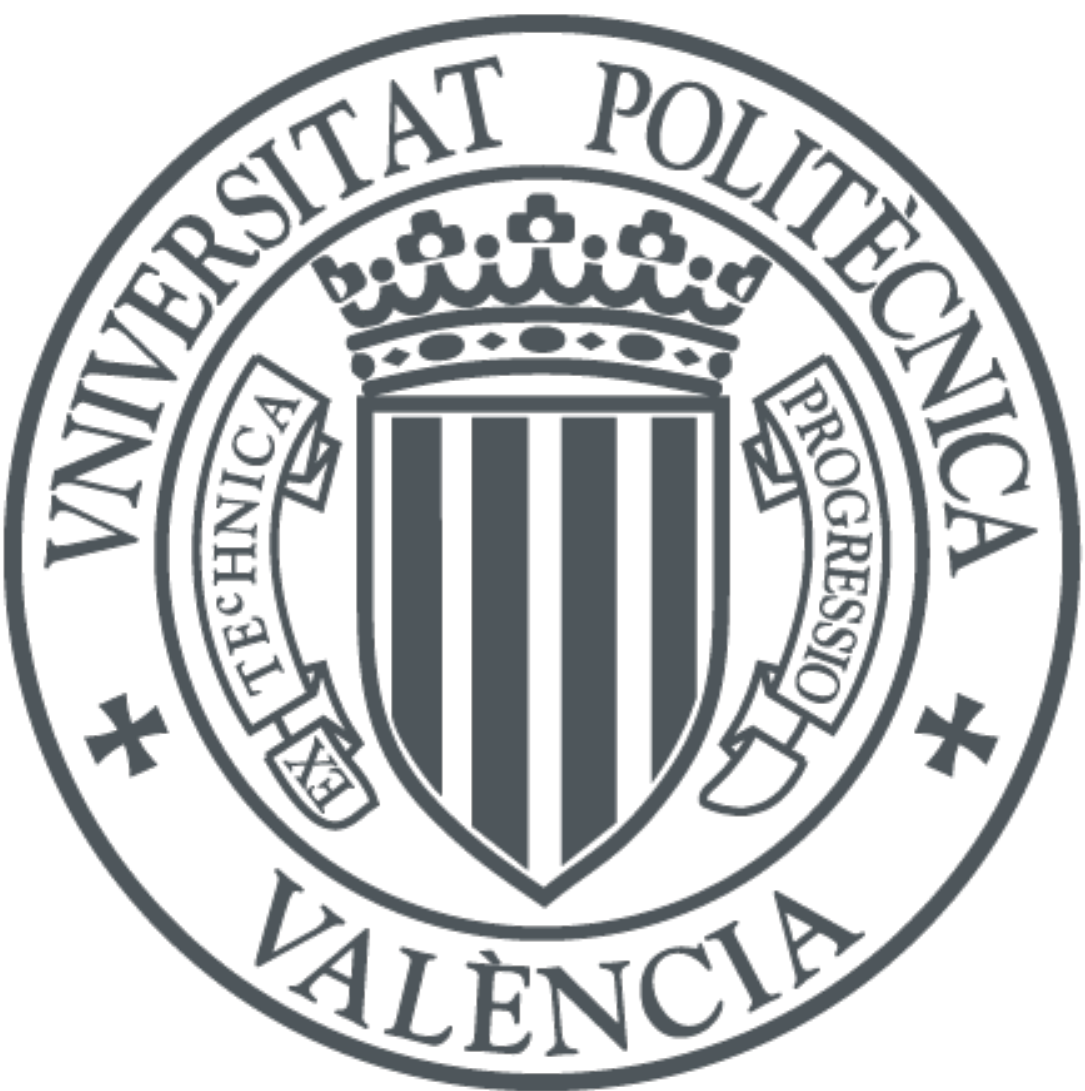

The final publication is available at

Copyright SAGE Publications (UK and US)

Additional Information 


\title{
Determination of the resonance response in an engine cylinder with a bowl-in-piston geometry by the finite element method for inferring the trapped mass
}

\author{
Alberto Broatch ${ }^{\mathrm{a}}$, Carlos Guardiola ${ }^{\mathrm{a}}$, Pau Bares $^{\mathrm{a}, *}$, Francisco D. Denia ${ }^{\mathrm{b}}$ \\ ${ }^{a}$ CMT-Motores Térmicos, Universitat Politècnica de València \\ ${ }^{b}$ Centro de Investigación de Tecnología de Vehículos, Universitat Politècnica de València
}

\begin{abstract}
Cylinder resonance phenomenon in reciprocating engines consists of high-frequency pressure oscillations excited by the combustion. The frequency of these oscillations is proportional to the speed of sound on pent-roof combustion chambers and henceforth the resonance frequency can be used to estimate the trapped mass, but in bowl-in-piston chambers a geometrical factor must be added in order to deal with the bowl disturbance.

This paper applies the finite element method to provide a resonance calibration for new design combustion chambers, which are commonly dominated by the bowl geometry near the top dead center. The resonance calibration does not need any sensor information when it is solved by a FEM procedure, and consequently, is free from measurement errors. The calibration is proven to be independent of the chamber conditions and the results obtained are compared with experimental data by using spectral techniques and measuring precisely the trapped mass.
\end{abstract}

Keywords: Trapped mass estimation, Pressure resonance, Combustion diagnosis, Finite Element Method

\footnotetext{
${ }^{*}$ Corresponding author

Email addresses: carguaga@mot.upv.es (Carlos Guardiola), pabamo@mot.upv.es (Pau Bares)
} 


\section{Nomenclature}

$\begin{array}{ll}A T D C & \text { After top dead center } \\ B P & \text { Burnt products } \\ C I & \text { Compression ignition } \\ E G R & \text { Exhaust gas recirculation } \\ F E M & \text { Finite element method } \\ H C C I & \text { Homogeneous charge compression ignition } \\ I V C & \text { Intake valve closing } \\ O P & \text { Operation point } \\ R C C I & \text { Reactivity controlled compression ignition } \\ S I & \text { Spark ignition } \\ S O C & \text { Start of combustion } \\ S T F T & \text { Short time Fourier transform } \\ T D C & \text { Top dead center } \\ U B P & \text { Unburnt products } \\ V V T & \text { Variable valve timing } \\ W D & \text { Wigner distribution }\end{array}$

\section{Introduction}

In-cylinder pressure analysis provides a valuable information of the combus5 tion process [? ? ]. The pressure signal contains a wealth of information and, depending on the application, a different part of the pressure signal must be processed, e.g. the compression stroke is used to determine the trapped air mass by $\Delta p$ methods [? ? ], the low frequency components are employed to estimate the wall heat transfer [? ], the frequency components above $500 \mathrm{~Hz}$

10 are correlated with the noise radiated [? ], and sudden changes at the pressure signal are used to detect the combustion [? ].

The understanding of the pressure signal and the different phenomena taking place in a piston engine is crucial for properly processing the signal. F. Payri

15 et al. analysed the frequency components of the in-cylinder pressure signal decomposing the signal by three different phenomena, namely pseudo-motored, combustion and resonance [? ]. Concretely, the resonance has been largely studied along decades because it is associated with knock, which is a limiting factor in SI engines [? ] and is a source of noise in CI engines [? ]. It consists

20 of a gas oscillation excited by abrupt changes in the in-cylinder pressure.

The problem of the resonance frequency evolution during the combustion event was firstly faced by Draper in 1938, by solving the wave equation with cylindrical boundary conditions. He ended up with a linear relation between the 25 resonance frequencies and the speed of sound [? ]. This relation was then used to estimate the resonance evolution as a function of the gas properties in order to filter the resonance [? ]. Hardly anyone tried the opposite process: detecting the resonance frequency in order to determine the gas properties. Hickling et al. 
used the pressure resonance to calculate the bulk temperature [? ], Bordisco et al. dedicated a section for the possible application of their statistical model of the resonance to the estimation of the trapped mass [? ] and finally Guardiola et al. developed a complete method for the trapped mass estimation by using STFT [? ].

The main trouble of the inverse process is that the cylindrical solution does not fit the response of new design chambers, which commonly dispose of complex bowl geometries for controlling the turbulence of the gasses [? ? ]. When the aim is locating the pressure resonance in order to filter it, the precision of the cylindrical solution is sufficient; however, when the pressure resonance must be

40 used to estimate gas variables, the accuracy of the estimation directly depends on the hypothesis assumed. Past solutions for the inverse process calibrated the chamber resonance response by using experimental data [? ? ]. But trusting the sensor measurements implies that the accuracy of the method is affected by the sensors precision, which normally have associated errors higher than $1 \%$ [? ].

Scholl et al. used the Finite Element Method (FEM) [? ] to verify the Draper's cylindrical correlation on a pent-roof combustion chamber [? ]. Herein FEM is used to permit obtaining a calibration of the bowl effect by analysing only the geometry. The final calibration, which relates the resonance frequencies

50 and the speed of sound, is demonstrated to be independent of the chamber conditions and is compared with experimental data extracted from a RCCI heavyduty 4 stroke engine. The next section is aimed to introduce the reader with the method to compute the trapped mass through the resonance phenomenon, an extended description of the FEM-based procedure is given in section 3, the

${ }_{55}$ experimental facilities employed are described in section 4, the results and the consequent discussion are exposed in section 5 , and finally the main contributions of the method are highlighted in section 6 .

\section{From resonance to trapped mass}

The cylindrical solution of the pressure resonance considered by Draper [? ] relates the speed of sound $(a)$ for a given crank angle $(\alpha)$ with the resonance frequencies $\left(f_{i, j}\right)$ as a function of the bore $(D)$ and a Bessel constant $\left(B_{i, j}\right)$ :

$$
f_{i, j}(\alpha)=\frac{a(\alpha) B_{i, j}}{\pi D}
$$

Note that the Bessel constant in equation (1) is related to the solution of the wave equation in a cylindrical domain [? ]. Table 1 shows the first values of the Bessel constant for the cylindrical solution considering rigid walls [? ].

Guardiola et al. inverted the problem [? ]: they detected the resonance

${ }_{65}$ frequency $\left(f_{i, j}\right)$ with STFT and computed the final mass $(m)$ by iterating (by bisection or fixed-point iteration) the following equation: 
Table 1: Bessel constants of the first resonance modes for a cylindrical chamber [? ]

$$
\begin{array}{rc}
\text { Mode } & B_{i, j} \\
\cline { 2 - 2 } 1,0 & 1.841 \\
2,0 & 3.054 \\
0,1 & 3.832 \\
3,0 & 4.201 \\
1,1 & 5.332 \\
& \\
f_{i, j}(\alpha, m)=\frac{B_{i, j} \sqrt{\gamma(\alpha, m) p(\alpha) V(\alpha)}}{\pi D \sqrt{m}}
\end{array}
$$

where $p(\alpha)$ is the low-frequency component of the pressure signal (below $3 \mathrm{kHz}$ ), $V(\alpha)$ is the instantaneous volume and $\gamma(\alpha, m)$ is the ratio of constant pressure and the constant volume heat capacities, which may be estimated using corre70 lations as in [?].

The Bessel constants, collected in table 1, have been accepted by many authors, some of them are gathered in the appendix of [? ]. However, the growing complexity of new combustion chambers invalidates the cylindrical approach 75 near the Top Dead Center (TDC).

Figure 1 shows the pressure signal distribution over the frequencies as a function of the cycle evolution and superposes the theoretical cylindrical resonance response (white line): the cylindrical response was calculated from equation 2

so by using the first asymmetric mode $\left(B_{1,0}=1.841\right)$ and by inferring the speed of sound on the basis of a correlation [? ] as a function of gas composition and temperature (obtained from the cylinder pressure, volume and trapped mass). In this figure the discrepancies near the TDC are easily perceptible, despite the convergence far from the TDC (where the bowl effect is negligible).

The solution proposed in references [? ? ] was based on assuming that the resonance frequencies can be linearly correlated with the speed of sound but only when the geometry is maintained unaltered. As a consequence, the Bessel constant $\left(B_{i, j}\right)$ must be experimentally calibrated for each crank angle position

$90\left(B_{i, j}^{c}(\alpha)\right)$. All the past works on the topic calibrated the Bessel constant relaying on sensor data (normally only the first mode is considered because it is the most representative):

$$
B_{1,0}^{c}=\frac{\pi D \sqrt{m} f_{1,0}}{\sqrt{\gamma p V}}
$$

Figure 2 is a scheme of the method implemented by Guardiola et al. (black solid arrows) for the determination of the trapped mass [? ]. Their calibration 95 procedure (grey dashed arrows) was based on experimental data, where both the experimental determination of the resonance frequencies and the estimation of 


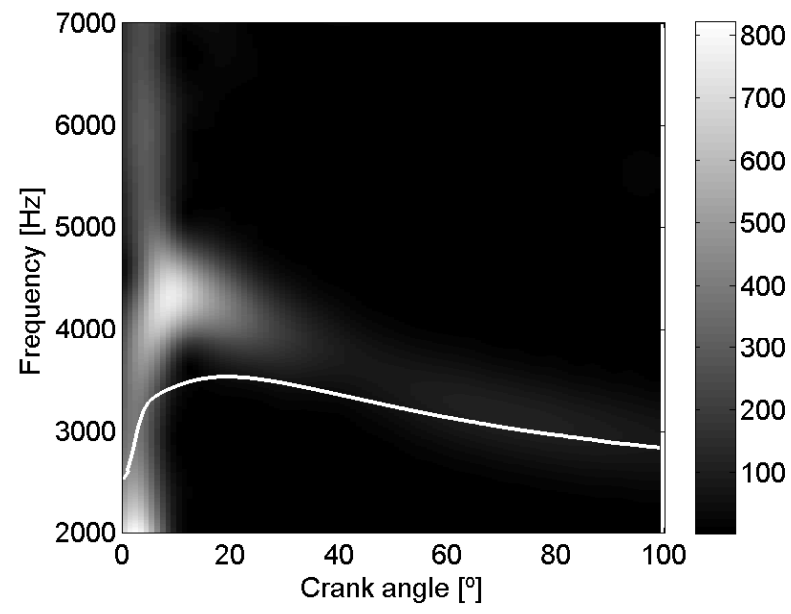

Figure 1: Theoretical Bessel constants (white line) over a Spectral distribution of a pressure signal (STFT)

the trapped mass are important sources of errors. The aim of the new calibration method is to obtain $B_{1,0}^{c}(\alpha)$ with no need of sensor data (grey solid arrow): using FEM, the final Bessel constant is obtained only as a function of the geometry.

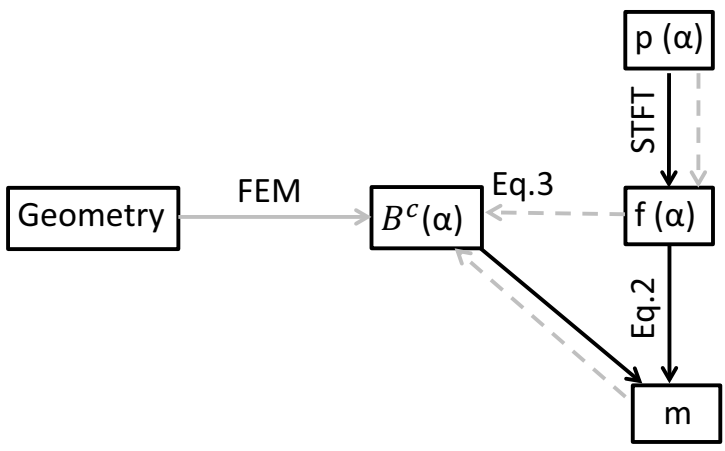

Figure 2: Scheme of the pressure resonance method

100

\section{Finite element model}

For a specific bowl geometry, the natural frequencies and the associated pressure modes can be computed through a FE-based eigenvalue problem [? ]. Figure 3 depicts a particular finite element discretization used in the computations for a crank angle $=10^{\circ}$. The first mode $(1,0)$ is also represented by 105 showing the pressure distribution in the figure for illustration purposes. In this 
particular case, an asymmetric behavior can be observed, with a diametrical nodal line (green) separating pressure regions in phase opposition.
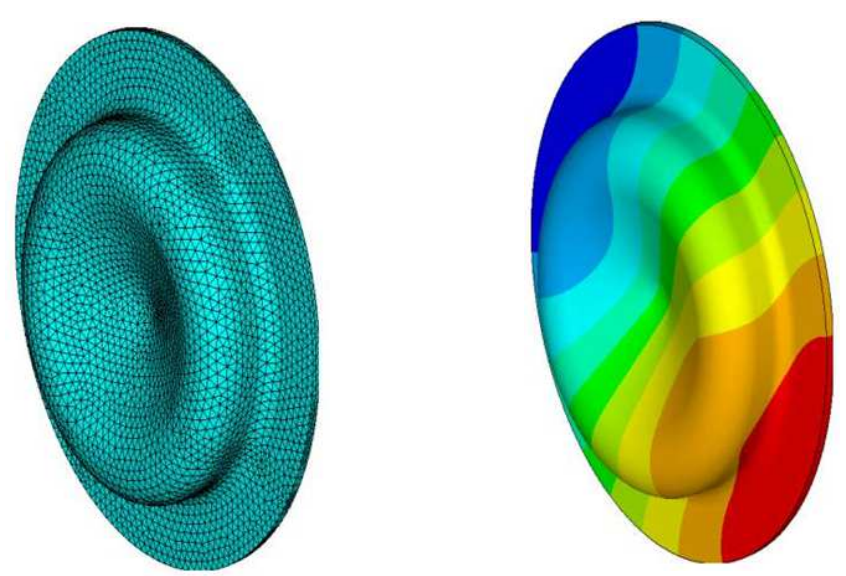

Figure 3: Finite element discretization and pressure mode $(1,0)$

The numerical calculations have been carried out with a refined FE mesh consisting of three-dimensional 10-node quadratic tetrahedral elements. To guarantee an accurate prediction, the final bowl discretization contains 115659 nodes and 78284 elements, whose approximate size is $0.0025 \mathrm{~m}$. This provides more than 65 quadratic elements per wavelength for the frequency associated with mode $(1,0)$. An approximate variation of $0.008 \%$ is found in the natural frequency $f_{1,0}$ between two consecutive meshes with a refinement ratio of 2 , from an element size of $0.005 \mathrm{~m}$ to $0.0025 \mathrm{~m} \mathrm{[?} \mathrm{].} \mathrm{Thus,} \mathrm{it} \mathrm{can} \mathrm{be} \mathrm{assumed} \mathrm{that} \mathrm{the}$ FE solution has reached the asymptotic range of convergence and the estimated frequency is sufficiently accurate for the current calibration procedure.

\section{Experimental data}

The results obtained from finite element computations have been compared with pressure data acquired on a single cylinder heavy-duty engine. The latter was equipped with gasoline port injection and diesel direct injection, allowing multiple diesel injections during the cycle. EGR facilitated the control of the engine over different combustion modes, while electrohydraulic variable valve timing (VVT) permitted controlling the valves to change the mass instantaneously [?].

A sketch of the bowl geometry is shown in figure 4 and the main parameters of the engine are summarized in table 2 . The geometry evolution was supposed to follow the mechanical equation: 


$$
V(\alpha)=V_{c c}+\frac{\pi D^{2}}{4}\left[l+r(1-\cos (\alpha))-\sqrt{l^{2}-r^{2} \sin (\alpha)^{2}}\right]
$$

where $l$ is the length of the rod, $r$ is the radius of the crank and $V_{c c}$ is the chamber volume at the TDC.

Table 2: Main engine characteristics

\begin{tabular}{lc}
\hline Displaced volume $\left(V_{d}\right)$ & $1806 \mathrm{cc}$ \\
Bore $(D)$ & $123.6 \mathrm{~mm}$ \\
Engine speed $(n)$ & $1200 \mathrm{rpm}$ \\
Combustion mode & RCCI-Diesel
\end{tabular}

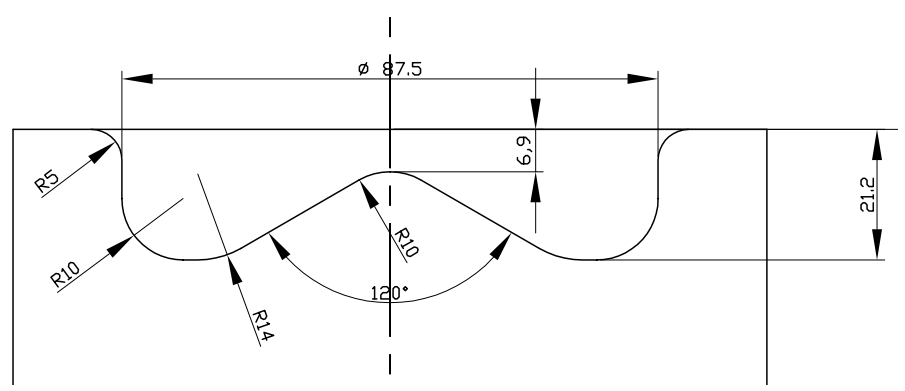

Figure 4: Bowl geometry (dimensions in $\mathrm{mm}$ )

A classical procedure combining several measurements and models was used to calculate the in-cylinder trapped mass and will be referred hereinafter as the auxiliary method.

The auxiliary method was based on the direct measurement of the air mass flow through hot film anemometry, the determination of EGR by means of $\mathrm{CO}_{2}$ balance in the intake manifold, the measurement of the blow-by using the orifice measuring principle, and the determination of the fuel mass flow by a fuel balance. In order to estimate the residual gas fraction and the short circuit mass flow, an emptying-and-filling model as in [? ? ? ] was used for solving the

145 flow through the engine valves. The gas properties $(\gamma$ and $R$ ) were computed by semi-empirical equations developed by Lapuerta et al. [? ], dividing the mixture in three components, namely fuel, air and burnt products and the combustion evolution was assumed to follow the Rassweiler and Withrow model [? ].

The pressure was obtained by a Kistler $6125 \mathrm{c}$ sensor and processed by a $\mathrm{Na}$ tional Instruments RT-PXI acquisition system. The RT-PXI permitted a crank angle based acquisition (5 samples/deg) and a time based acquisition (up to 4 $\mathrm{MHz}$ ), both of them with 16 bits resolution. The crank angle based acquisition 
enabled locating the samples in order to phase it properly with the chamber volume while the time based acquisition permitted computing the actual resonance frequencies. The time between crank angle samples was recorded to compute the instantaneous engine speed and evaluate the differences between crank angle and time based acquisitions.

\subsection{Time-frequency analysis}

In order to obtain the frequency components with time resolution a spectral analysis should be performed. Most of the authors employ the Short Time Fourier Transform (STFT) because this permits a robust estimation of the spectrum with no important computational costs [? ]. In contrast to the Short Time

${ }_{165}$ Fourier Transform (STFT), which dilutes the frequency content due to the window effect [? ], the Wigner Distribution (WD) obtains the actual frequency content at the expense of a higher computational time and the creation of cross terms, also known as ghost terms [?].

${ }_{170}$ Although there are not big differences between WD and STFT, WD results have been added on detailed table information for more precise validation. All plots and figures where experimental frequency results have been represented were computed by STFT, because is the most extended time-frequency analysis tool. In this paper a blackman-harris window of $25^{\circ}$ was used for the STFT,

175 the WD computations were made with a resolution of $16 \mathrm{~Hz}$ (approximately 0.5 $\%$ of the resonance value) avoiding aliasing troubles [? ] and the cross terms were mitigated by pass-band filtering the pressure signal between $2 \mathrm{kHz}$ and 7 $\mathrm{kHz}$.

\section{Results and discussion}

The method was validated by computing different FEM simulations and by processing specific experimental data. It must be said that in order to calculate the Bessel constants from the pressure resonance frequency experimentally obtained the trapped mass should be carefully calculated (the auxiliary method stated at section 3 was used).

\subsection{Proportionality between speed of sound and resonance frequency}

Firstly, for a given geometry (or a given crank angle position) the Bessel constant $(f \pi D / a)$ was computed from different chamber conditions. The Bessel constant obtained should be only a function of the geometry if the hypothesis is met, i.e. for a given geometry the speed of sound is proportional to the resonance frequency.

On one hand bulk temperature variations were achieved by changing the load (from $25 \%$ to $75 \%$ ) and the SOC (from -5.4 to 14.5 CAD-ATDC) to check 
out experimentally the base hypothesis: 100 cycles were recorded at each OP and the resonance frequency was computed at each cycle. Table 3 shows detailed information at different crank angle position (25, 45 and 65 degrees after TDC), showing the mean and the standard deviation for the Bessel constants obtained for the 100 cycles. Figure 5 plots the results for different OP with bulk temperature values contained between the two points shown at table 3

Table 3: Experimental results of $f \pi D / a$ over different OP

\begin{tabular}{ccc|cc|cc} 
& & & STFT & WD & \\
\hline CA $[\alpha]$ & $\mathrm{a}[\mathrm{m} / \mathrm{s}]$ & Increment & $\bar{B}$ & $\sigma(B)$ & $\bar{B}$ & $\sigma(B)$ \\
\hline 25 & 740.41 & - & 2.085 & 0.006 & 2.075 & 0.008 \\
& 774.73 & $+4.6 \%$ & 2.090 & 0.01 & 2.076 & 0.015 \\
\hline 45 & 683.87 & - & 1.941 & 0.009 & 1.957 & 0.007 \\
& 726.98 & $+6.3 \%$ & 1.944 & 0.009 & 1.975 & 0.024 \\
\hline 65 & 637.10 & - & 1.891 & 0.009 & 1.908 & 0.007 \\
& 681.27 & $+6.93 \%$ & 1.882 & 0.01 & 1.898 & 0.01
\end{tabular}

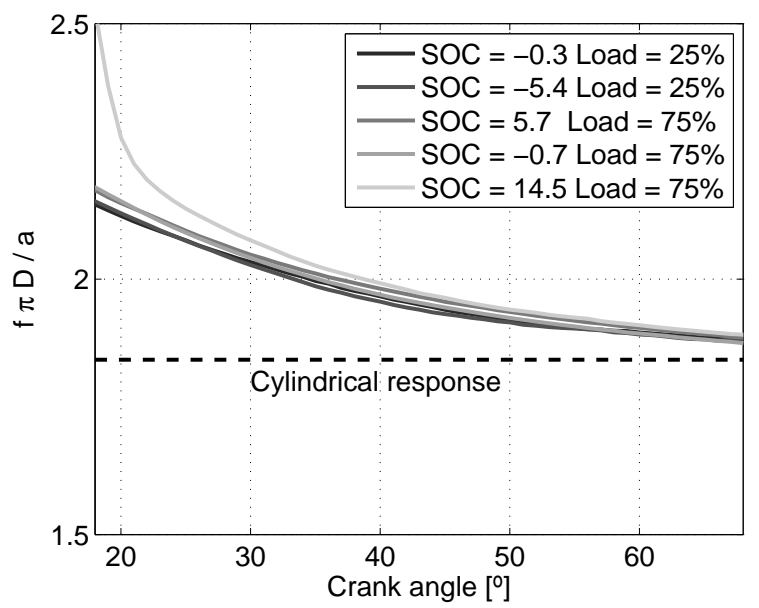

Figure 5: Experimental Bessel constants obtained for different OP (All SOC values in CADTDC)

It can be seen that despite the large variations of the speed of sound, the resonance frequencies varied proportionally, maintaining the coefficient $f \pi D / a$ almost constant at each crank angle position, which perfectly accords the base hypothesis. In figure 5 the OP starting combustion at 14.5 CAD-TDC does not coincide because of the late combustion.

On the other hand the results of FEM simulations over different in-cylinder chamber conditions $(a=\sqrt{\gamma R T})$, at two different crank angle positions (5 and 
30 degrees after TDC) are provided in table 4 in order to check out the consistency of the method.

\begin{tabular}{ccccc}
\multicolumn{5}{c}{ Table 4: FEM results over different chamber conditions } \\
CA $[\alpha]$ & a $[\mathrm{m} / \mathrm{s}]$ & Increment & $f_{1,0}[\mathrm{~Hz}]$ & $f \pi D / a[-]$ \\
\hline 5 & 852.1 & - & 5057.8 & 2.29362 \\
& 766.9 & $-10 \%$ & 4552.0 & 2.29365 \\
& 937.3 & $+10 \%$ & 5563.6 & 2.29364 \\
\hline 30 & 786.8 & - & 4125.2 & 2.02599 \\
& 708.1 & $-10 \%$ & 3712.7 & 2.02601 \\
& 865.5 & $+10 \%$ & 4537.7 & 2.02598
\end{tabular}

\subsection{Validation with experimental data}

The calibration obtained by FEM was compared with experimental data extracted from specific tests, where the trapped mass was measured by the auxiliary method and the pressure was acquired at $1 \mathrm{MHz}$. Figure 6 compares the FEM calibration with experimental data of 100 consecutive cycles over 4 different OP (400 cycles with different chamber conditions). In order to appreciate the variations between the 400 cycles the standard deviation was computed as:

$$
\sigma=\sqrt{\frac{\sum_{i=1}^{N}\left(x_{i}-\bar{x}\right)^{2}}{N-1}}
$$

multiplied by three and represented with a gray dashed line around the averaged 215 value. Dashed line in the figure, labeled with A, indicates the end of the combustion. As it may be appreciated, there is very good agreement between the FEM results and the experimentally determined Bessel constant in the region far from the TDC, specially when the combustion has finished. However, when approaching the combustion phase there is a significant shift in the measured value. Next section discuss if it could be rooted on the existence of temperature non homogeneities, thus violating the calculation assumptions.

\subsection{Effect of temperature gradients across the combustion chamber}

High temperature gradients could disturb the pressure acoustical response. 225 Although HCCI combustion is not especially affected because of the short duration and the nearby homogeneous combustion, high temperature gradients are expected on classical CI due to diffusive combustion.

In order to discern the effects of temperature gradients in combustion chambers a 2-zones finite element model was designed dividing the mixture into two species, namely burnt products and unburnt products, such as sketched in figure 7 . The simulated geometry was maintained constant $\left(30^{\circ}\right.$ after TDC) to 


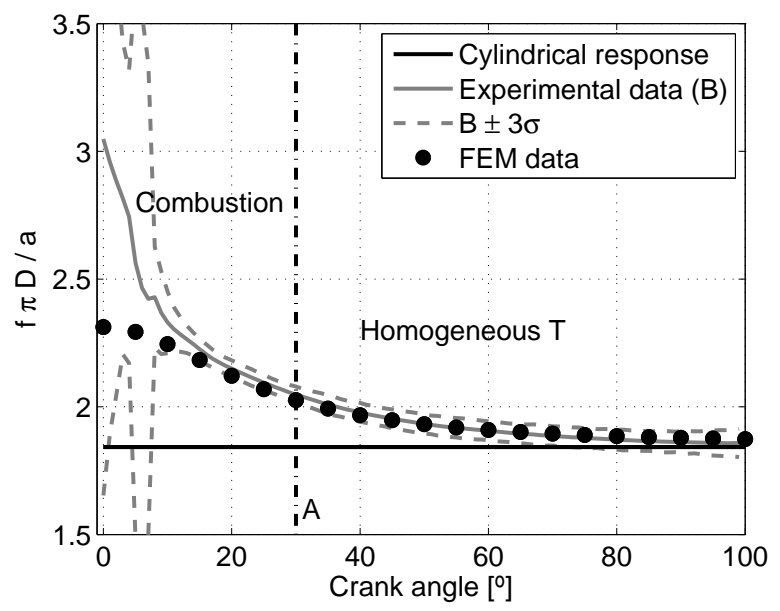

Figure 6: Final calibration (big black dots) over averaged experimental results of 400 cycles

separate the effect of the geometry with the temperature distribution. The temperature distribution modeled does not intend to simulate a real combustion but only the qualitative effect of temperature variations on the combustion chamber.

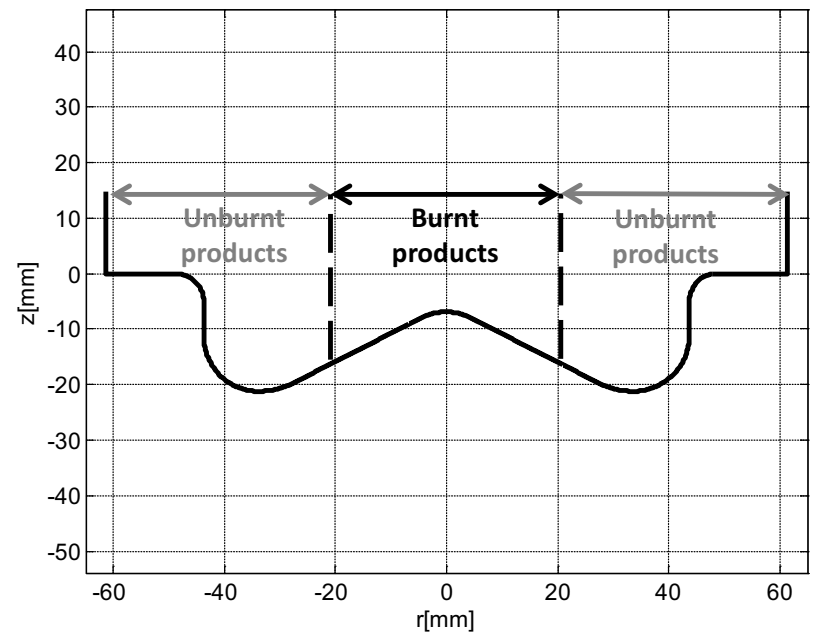

Figure 7: Two zones model sketch

Real data from an experimental test at $25 \%$ load was extracted to determine the combustion evolution and the energy delivered. The energy delivered was used to calculate the bulk temperature of the simulation by assuming constant volume and using the first law of thermodynamics. 


$$
d Q=m C_{v} d T+p d V=m C_{v} d T
$$

Equation 6 determines the bulk temperature estimated for the constant geometry chamber. The pressure and the gas constant $(R)$ were assumed constant along the chamber. As a consequence, mass, volume and temperature are related by equation 7 derived from ideal gas law. Figure 8 shows the final temperatures simulated.

$$
\left(\frac{V}{m T}\right)_{B P}=\left(\frac{V}{m T}\right)_{U B P}=\left(\frac{V}{m T}\right)_{A L L}
$$

Although crank angle evolution was maintained in figures 8 and 9 and in table 5 it does not represent a variation in geometry but only a more familiar measure of time evolution (at constant engine speed).

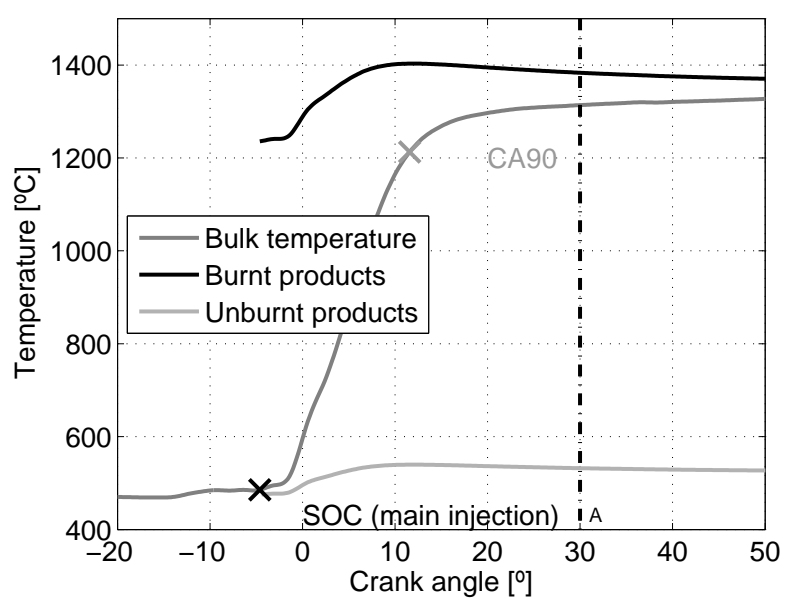

Figure 8: Temperature profile simulated

Table 5 shows the results at different moments of the combustion (1, 2.4, 20.3, 73.4 and $91.4 \% \mathrm{MFB}$ ), the temperature was modelled by assuming uni255 form bulk temperature $(1 z)$ and by dividing the chamber on burnt and unburnt products $(2 z)$. It is evidenced that temperature inhomogeneities along the chamber perturb the acoustical response: by assuming uniform bulk temperature a resonance frequency lower than by assuming temperature variations is always obtained (with differences up to $11.4 \%$ ). Low differences are located at the 260 beginning of the combustion (where almost all the mixture is unburnt) and at the end of the combustion (where almost all the mixture is burnt), a line named 


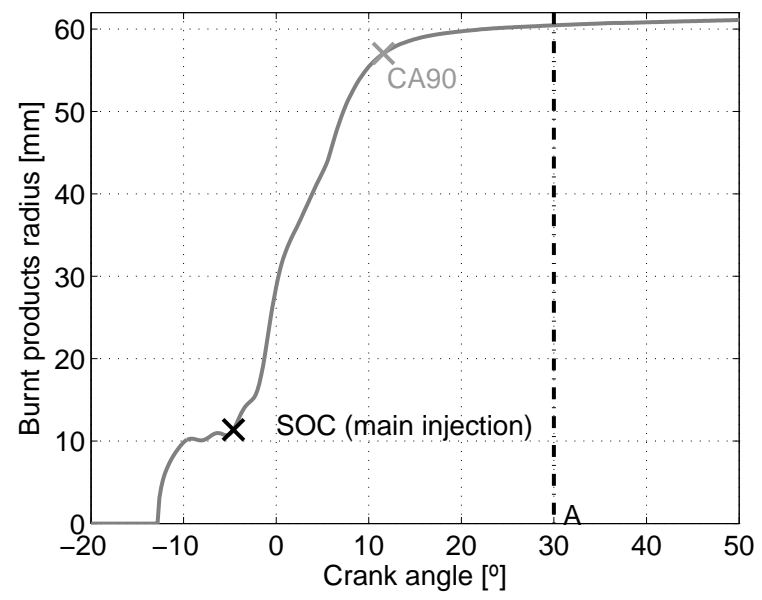

Figure 9: Burnt products radius evolution during combustion

" $A$ " was represented on figures 6,8 and 9 to locate where the temperature inhomogeneities become negligible.

Table 5: FEM results over temperature chamber variations

\begin{tabular}{ccccc} 
CA $[\alpha]$ & MFB $[\%]$ & $f_{1,0}^{1 z}[\mathrm{~Hz}]$ & $f_{1,0}^{2 z}$ & Difference $[\%]$ \\
\hline-12 & 1 & 2295.9 & 2306.8 & 0.47 \\
-2.2 & 2.4 & 2346.6 & 2462.1 & 4.69 \\
1.4 & 20.3 & 2729.0 & 3081.6 & 11.44 \\
10 & 73.4 & 3585.8 & 3857.4 & 7.04 \\
24 & 91.4 & 3802.0 & 3899.2 & 2.49
\end{tabular}

Results shown in Table 5 could suggest that the applicability of the resonance frequency method to conventional diesel engines is compromised, since in conventional diffusive combustions the temperature gradient will be significantly higher than that found in the RCCI engine used in [? ]. However, results summarized in Table 5 are for an infinite gradient with two zones of different 270 temperature, which corresponds to an extreme case. Additionally, if the method is applied in a window excluding the vicinity of the top dead center (i.e. during the expansion stroke after the combustion has ended and the burnt gases are sufficiently mixed with the surrounding air), temperature in the cylinder approaches that of a single-zone homogeneous mix, and the assumptions in (1)-(3)

275 are valid. Note that even in the case of RCCI engines, the method may not be applied during the combustion itself, as it is derived from Figure 5.

The reader will find in Appendix A an experimental validation on a CI 2.0 liter engine, which illustrates the applicability of the resonance frequency 
280 method for inferring the cylinder charge in the case of conventional CI engines if the analysis window is adequately selected.

\section{Conclusions}

A FEM-based procedure has been developed to calibrate the Bessel constant. This calibration procedure is the final tool for implementing a new method to 285 compute the trapped mass through the pressure resonance, which has been recently developed by the authors [? ]. In the authors' opinion the FEMbased approach presents many advantages in comparison with an experimental calibration:

- Using FEM instead of an experimental calibration ensures that the method does not rely on sensor data for finding the Bessel constant and consequently eliminates one important source of errors at the final trapped mass measurement.

- When using the pressure signal at the calibration, the phasing of the different signals becomes crucial, being the location of the TDC a major issue.

- FEM permits avoiding spectral methods, which have lots of associated problems: aliasing, computational time, discretization errors, ghost terms, window influence, etc.

Nevertheless, FEM simulations with a two zones model show that temperature gradients strongly influence on the acoustic response. As consequence, the results obtained are only valid when combustion is finished.

The calibration was made over a 4 stroke heavy duty RCCI engine, with a bowl geometry described in figure 4. The final Bessel constant was obtained ev-

305 ery 5 degrees between the TDC and 100 degrees (after TDC and before TDC). Finally the FEM calibration was validated with an excellent agreement with experimental data.

\section{Acknowledgement}

This research has been partially sponsored by the European Union in framework of the POWERFUL project, seventh framework program FP7/20072013, theme 7, sustainable surface transport, grant agreement No. SCP8-GA-2009234032. The authors gratefully appreciate this support. 


\section{Appendix A: Evaluation of the method}

Originally, the method was validated by Guardiola et al. over a RCCI combustion [? ]. Figure 10 compares the results obtained by the resonance method and by the auxiliary reference method over 54 operation points; trapped air mass ranged from $2900 \mathrm{mg} / \mathrm{str}$ to $8000 \mathrm{mg} / \mathrm{str}$. Error was calculated as:

$$
\operatorname{Error}[\%]=\frac{\left|m_{\text {aux }}-m_{\text {res }}\right|}{m_{\text {aux }}} \cdot 100 \%
$$

where $m_{\text {aux }}$ is the trapped mass estimated by the auxiliary method and $m_{\text {res }}$ represents the trapped mass obtained by the resonance method. The mean averaged error for this engine and data set was below $2 \%$.

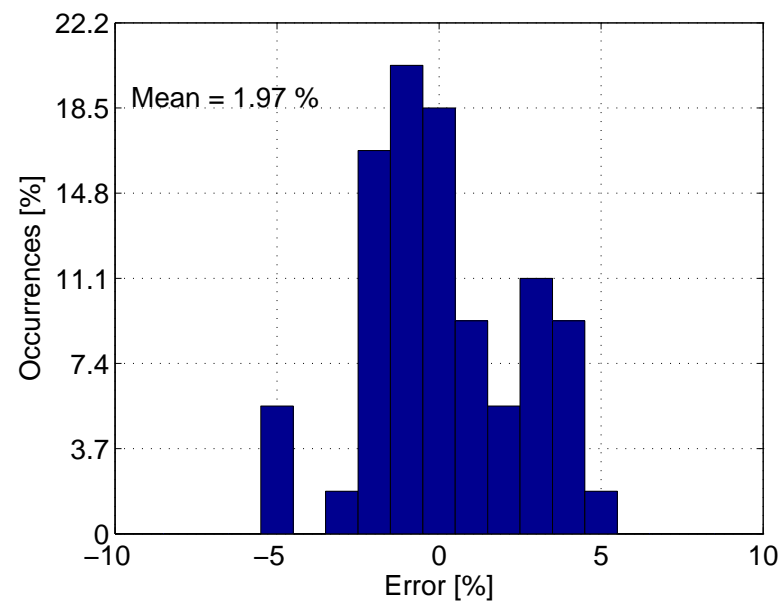

Figure 10: Evaluation of the resonance method on a RCCI engine

The good agreement with the data plotted was used in [? ] to validate the method for such engine, characterized by a fast and homogeneous combustion. However, FEM results shown in the present paper suggest that the method could be unusable in the case of classical CI engines, due to the existence of important temperature gradients. For validating the method over a CI combustion the method was run on a 4 stroke CI engine equipped with the necessary sensors for applying the auxiliary method (air mass flow, EGR measurement through $\mathrm{CO}_{2}$ balance and fuel mass flow). 808 tests were run covering from 1000 to $3000 \mathrm{rpm}$, and from idle to full load. In order to avoid the interference of the combustion, the method was applied on the expansion stroke with a window from $45^{\circ}$ to $85^{\circ}$ ATDC).

Figure 11 shows a histogram of the difference of the resonance method with regards to the auxiliary method for the tested CI engine. When comparing 
figures 10 and 11 it may be noticed that the averaged error is similar in both engines (around $2 \%$ ) and the resonance method does not usually differ more than $5 \%$ with the auxiliary method. It must be noticed that the auxiliary method do not represent the actual trapped mass and the error associated with the method (sensors and models) is estimated to be below $5 \%$ in [? ]. Consequently, it is not possible to discern the source of the differences between the two methods reported in the histograms in figures 10 and 11.

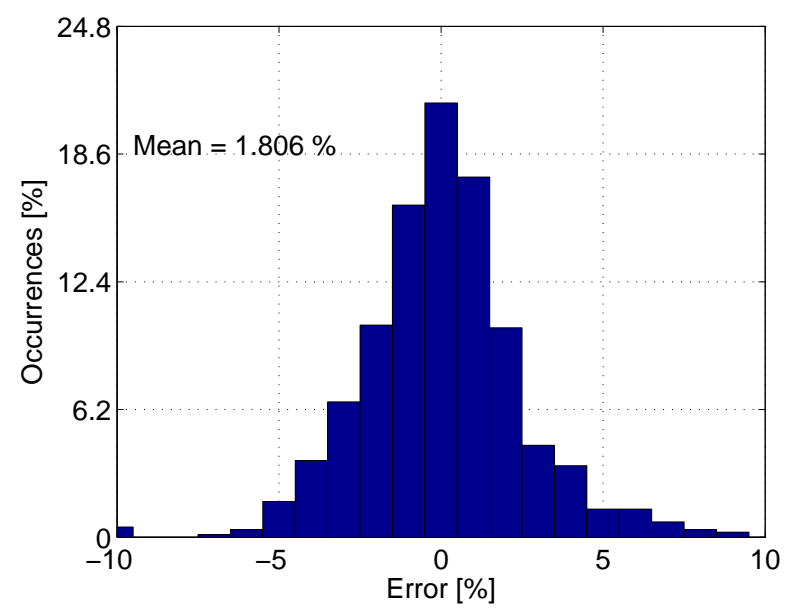

Figure 11: Evaluation of the resonance method on a CI engine 